\title{
Diffusion MRI Connections in the Octopus Brain
}

\author{
Russell E. Jacobs* \\ Department of Physiology and Neuroscience, Zilkha Neurogenetics Institute, Keck School of Medicine of USC, \\ Los Angeles, CA 90089-2821, USA
}

\begin{abstract}
Using high angle resolution diffusion magnetic resonance imaging (HARDI) with fiber tractography analysis we map out a meso-scale connectome of the Octopus bimaculoides brain. The brain of this cephalopod has a qualitatively different organization than that of vertebrates, yet it exhibits complex behavior, an elaborate sensory system and high cognitive abilities. Over the last 60 years wide ranging and detailed studies of octopus brain anatomy have been undertaken, including classical histological sectioning/staining, electron microscopy and neuronal tract tracing with injected dyes. These studies have elucidated many neuronal connections within and among anatomical structures. Diffusion MRI based tractography utilizes a qualitatively different method of tracing connections within the brain and offers facile three-dimensional images of anatomy and connections that can be quantitatively analyzed. Twenty-five separate lobes of the brain were segmented in the 3D MR images of each of three samples, including all five sub-structures in the vertical lobe. These parcellations were used to assay fiber tracings between lobes. The connectivity matrix constructed from diffusion MRI data was largely in agreement with that assembled from earlier studies. The one major difference was that connections between the vertical lobe and more basal supra-esophageal structures present in the literature were not found by MRI. In all, 92 connections between the 25 different lobes were noted by diffusion MRI: 53 between supra-esophageal lobes and 26 between the optic lobes and other structures. These represent the beginnings of a mesoscale connectome of the octopus brain.
\end{abstract}

Key words: Octopus, Cephalopod, Diffusion MRI, Connectome, Neuroimaging

\section{INTRODUCTION}

Cephalopod ancestors arose around 530 million years ago (mya) and during the mid-Palaeozoic ( 416 mya) cephalopods diverged into nautiloids and the presently dominant coleoids (e.g., squids, cuttlefish and octopods) [1,2]. The coleoid cephalopods are among the most neurologically complex invertebrates with Octopodiformes being perhaps the most complex and intensively studied [3-9]. Octopus behavior is complex and multifaceted [4-6, $10,11]$, offering a unique system for studying the neuronal basis of learning and memory [7, 12-16]. The genome of Octopus bimaculoides was sequenced in 2015 with interesting similarities with the

Submitted November 23, 2021, Revised February 15, 2022,

Accepted February 18, 2022

* To whom correspondence should be addressed.

TEL: 1-323-865-1668, FAX: 1-626-585-1505

e-mail: jacobsr@usc.edu vertebrate (e.g., massive expansion of the protocadherins - important in neuronal development) [17]. Recent evidence indicates that at least one neurotransmitter system (the serotonergic) is conserved in octopus [18]. Curiously, the octopus displays a high level of RNA editing; a post-transcriptional process quite uncommon across a wide variety of species: human, drosophila \& C. elegans $[19,20]$. Thus, the octopus is a compelling system for comparison with mammals due to its ancient origin and great anatomical differences combined with fascinating similarities in problem solving skills, learning and memory $[11,21]$

Following the pioneering work of Young [22], a large number of anatomical and physiological studies of the octopus and related coleoid cephalopods have been undertaken. In addition to detailed histological studies [8, 12, 15, 16, 23-36], other efforts have utilized computed tomography $[37,38]$, electron microscopy [39, 40], positron emission tomography [41], fluorescent dye labeling of neuronal connections [42-44] and magnetic resonance imaging [45-48].
Copyright $\odot$ Experimental Neurobiology 2022. www.enjournal.org
This is an Open Access article distributed under the terms of the Creative Commons Attribution Non-Commercial License (http://creativecommons.org/licenses/by-nc/4.0) which permits unrestricted non-commercial use, distribution, and reproduction in any medium, provided the original work is properly cited. 
Recently Jung et al. [25] have performed serial sagittal and coronal sections stained with Hematoxylin and Eosin (H\&E) of whole brains of the Long Armed Octopus, Octopus minor. They have identified and annotated more than 70 distinct anatomical regions providing a detailed atlas of this adult octopus. Chung et al. have married histological staining techniques with high resolution diffusion weighted MRI to create a meso-scale atlas of Sepioteuthis lessoniana brain including 426 connections between 23 different structures - a connectome of the squid brain [45] along the lines of similar work in the mouse $[49,50]$.

The octopus brain consists of some 30 differentiated lobes arranged in a circumesophageal Bauplan. Although the nervous system is composed of some half a billions neurons, over half are found in the ganglia of the eight arms $[36,51]$. While much is known about the octopus brain anatomy [22, 25, 33, 40, 52-54], there is a paucity of neuroimaging information about connectivity of regions that would lead to information about functional organization and circuitry underpinnings behavior. In this work we employ high resolution magnetic resonance imaging (MRI) to construct a mesoscale digital three-dimensional brain atlas of the adult Octopus bimaculoides, including assessment of connectivity between anatomic regions.

\section{MATERIALS AND METHODS}

\section{Sample collection and preparation}

Two male and one female adult specimens were collected off the Southern California coast by Aquatic Research Consultants (San Pedro, CA 90732, Fishes4Study.com, Chuck Winkler) and transported to USC the same day. Euthanasia was accomplished by immersion in a 1:3 mixture of isotonic magnesium chloride in artificial sea water. Specimen was maintained in this solution for at least 10 minutes after cessation of breathing, spontaneous behavior and motion of any kind before removing $[55,56]$. Specimen was transferred to $4 \%$ paraformaldehyde solution with stirring at $10^{\circ} \mathrm{C}$ for several days for fixation. Samples were soaked in $2.5 \mathrm{mM}$ Gadoteridol (Gd-HP-DO3A, Bracco Diagnostics Inc., Princeton, NJ) for 3 days. Samples were immersed in Galden (TMC Industries, MN) while being scanned.

\section{MRI}

All images were recorded with a MRSolutions (Guildford, Surrey, GU3 1LR, UK) PowerScan Preclinical MRI System 7.0T horizontal $24 \mathrm{~cm}$ bore scanner using a $35 \mathrm{~mm}$ diameter quadrature birdcage RF coil. It is equipped with two independent broadband RF transmit channels for excitation and decoupling, four independent broadband RF receive channels and a water cooled shielded gradi- ent coil set with integral second order shim coils.

Images were collected from 3 samples with a spin echo DWI sequence. A High Angular Resolution Diffusion Imaging (HARDI) scheme was used $[57,58]$ with 32 directions and 3 or 4 shells with b-values 500 2,000 s/mm $\mathrm{mm}^{2}$ and $4 \mathrm{~b}=0$ scans, TR/TE=400 ms/22 $\mathrm{ms}$ and isotropic resolution from $0.1 \mathrm{~mm}$ to $0.14 \mathrm{~mm}$. Directions \& shells from Caruyer et al. [59] (http://www.emmanuelcaruyer. com/q-space-sampling.php) using a distinct optimized spherically even distribution of gradient directions.

\section{Image analysis}

The reconstruction, fiber tracking, connectivity matrix and graph theoretical analysis were conducted using DSI Studio (http:// dsi-studio.labsolver.org). The restricted diffusion was quantified using restricted diffusion imaging [60]. The diffusion data were reconstructed using generalized q-sampling imaging [61] with a diffusion sampling length ratio of 1 . A deterministic fiber tracking algorithm [62] was used with the seeding region placed in the whole brain, the anisotropy threshold set to eliminate background voxels (0.022 0.034), the angular threshold set to 60 degrees and the step size set to $0.06 \mathrm{~mm}$. The fiber trajectories were smoothed by averaging the propagation direction with $20 \%$ of the previous direction. Tracks with length shorter than $0.5 \mathrm{~mm}$ or longer than $30 \mathrm{~mm}$ were discarded. A total of 2 seeds per voxel were placed in delineated regions of interest. Anatomical regions of interest (ROIs) were used as the brain parcellation and the connectivity matrix was calculated using the count of the connecting tracks. Anatomical ROIs were determined manually from the nqa and iso images.

For comparison of connectivity across samples, the count of connecting streamlines in the optic tract was set equal to 10000 . Following Chung et al. [45] we define a Connectivity Strength Index (CSI) for each ROI as the median of $\log _{10}$ (count of connecting tracts) of the 3 samples with a lower bound of 1.5.

Visualization was done with JColorGrid [63], chordiag [64], MRIcro [65] and ImageJ [66].

\section{RESULTS \& DISCUSSION}

$\mathrm{T}_{2}$ weighted MR images recapitulate much of the anatomic detail seen in histologically based cephalopod atlases and earlier work $[22,25,36,51,67]$, although at mesoscale $(125 \mu \mathrm{m})$ rather than microscopic scale. Fig. 1 shows orthogonal slices from one sample with a dozen distinct structures noted.

Isotropic diffusion (iso) and normalized quantitative anisotropy (nqa) [62] images derived from the HARDI analysis afforded more detail, thus better differentiation of more structures than were observed in $\mathrm{T}_{2}$ weighted images. Fig. 2 shows orthogonal iso 


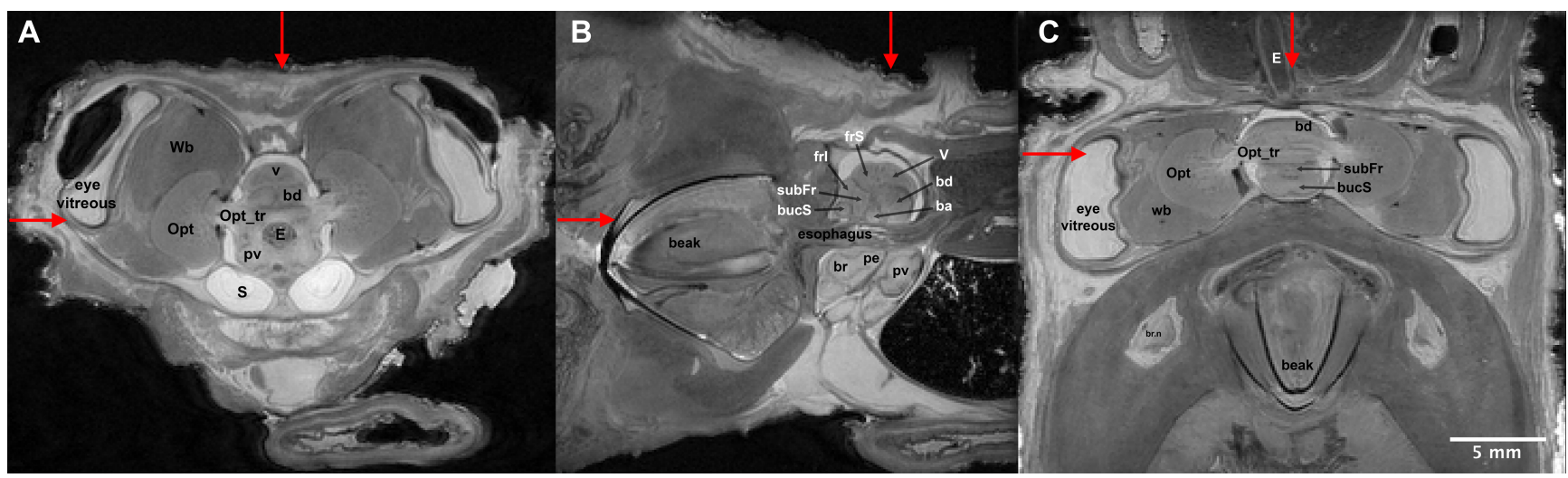

Fig. 1. Neuroanatomy from MR images. (A C) Transverse, sagittal \& coronal sections. Red arrows note location of orthogonal sections. Individual brain regions easily identified due to their encapsulation. Internal structure in optic lobes and filamentous optic nerves extending from the optic bulbs to the retinas are apparent. Abbreviations for brain structures: v_ll, vertical lobe lateral left; v_l, vertical lobe mediolateral left; v-mid, vertical lobe medial; v_r, vertical lobe mediolateral right; v-rr, vertical lobe lateral right; subV, sub vertical lobe; frS, superior frontal lobe; frI, inferior frontal lobe; subFr, subfrontal lobe; bucS, superior buccal lobe; bucP, posterior buccal lobe; ba, anterior basal lobe; bd, dorsal basal lobe; ped-r, peduncle lobe right; n.opt-R, optic nerves right; Opt-R, optic lobe right; gOpt-R, optic gland right; Opt_tr, optic tract; gOpt-L, optic gland left; Opt-L, optic lobe left; n.opt-L, optic nerves left; pedL, peduncle left; pv, pallioviseral lobe; pe, pedal lobe; br, brachial lobe.
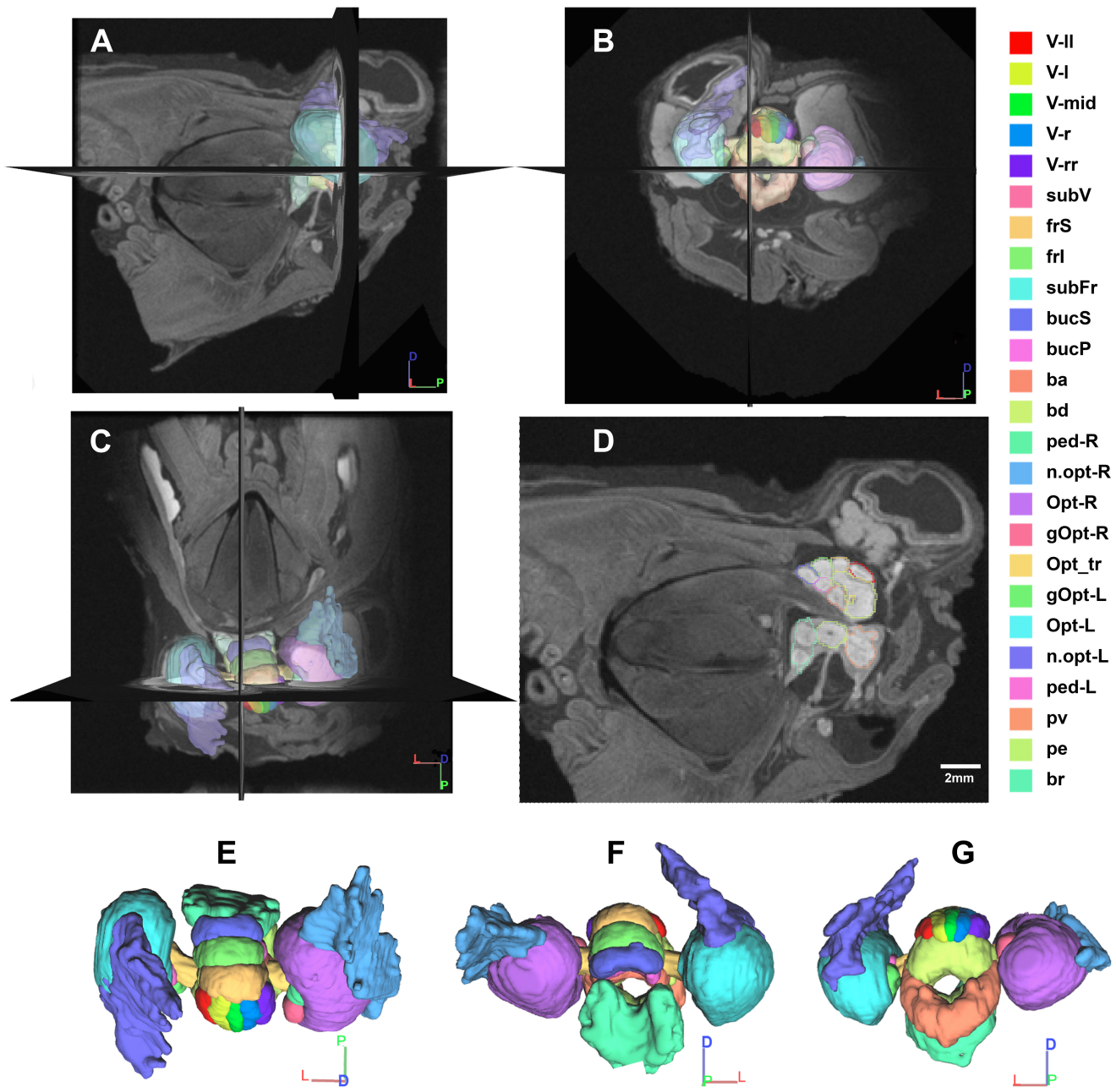

Fig. 2. Three Dimensional Anatomic Annotation. (A C) Three slices from isotropic diffusion weighted images (iso in DSI software) with semitransparent color-coded anatomy. (D) parasagittal slice with outlined anatomy used in the connectivity analysis. (E G) dorsal, anterior \& posterior views, respectively, of $3 \mathrm{D}$ surface rendering of anatomical regions. Coordinate system: D, dorsal-ventral; P, anteriorposterior; L, left-right. Abbreviations as in Fig. 1. 


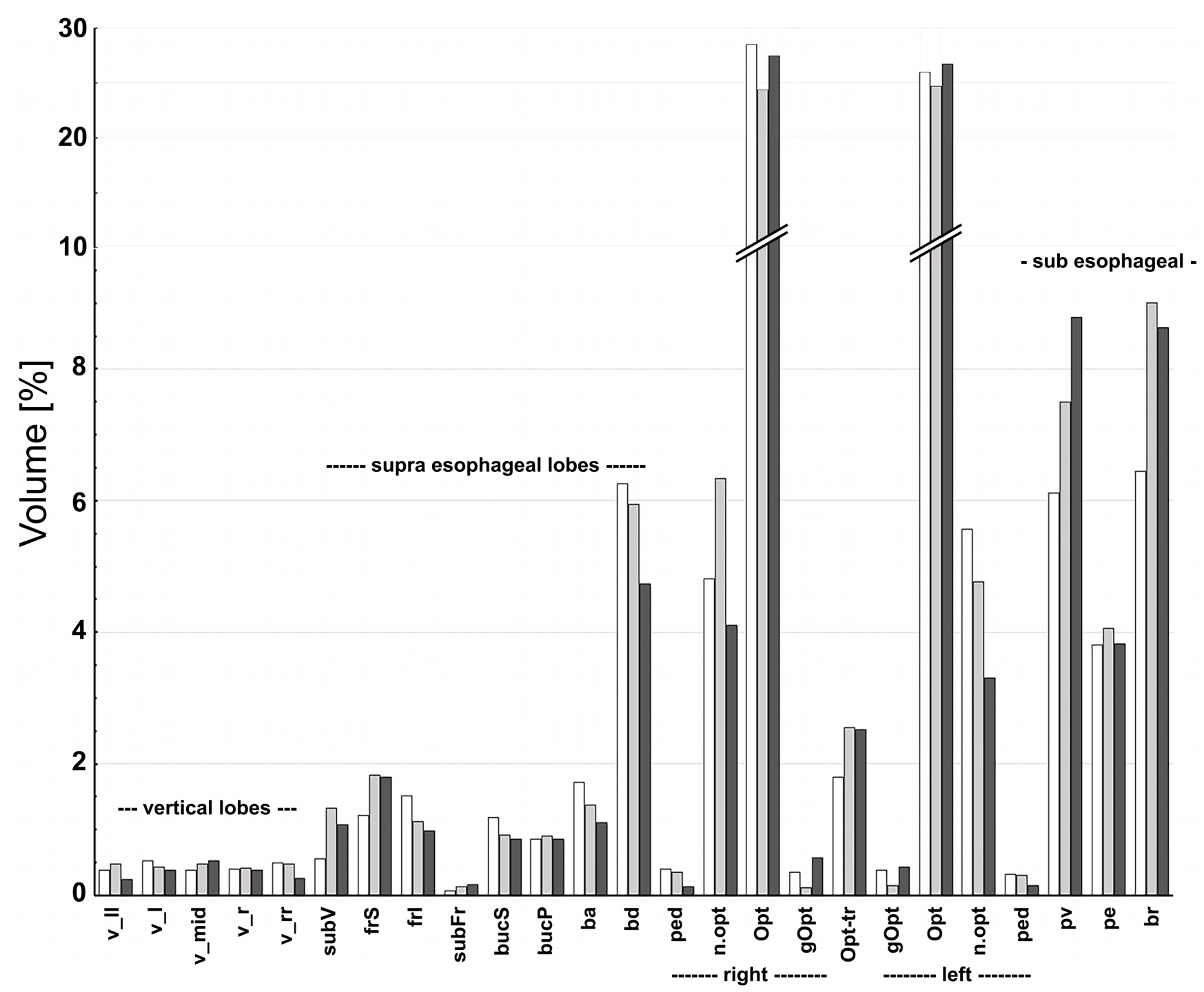

Fig. 3. Relative volumes of different anatomical regions. Relative volumes of all anatomical regions are consistent across 3 similar size samples. Abbreviations as in Fig. 1.

image slices with 25 anatomical structures, including all five subregions of the vertical lobe, shown as semitransparent 3D overlays on the gray scale slice (Fig. 2A 2C), a parasagittal slice with color shaded anatomy (Fig. 2D) and surface renderings of 3D annotated anatomy (Fig. 2E 2G). All annotations made with reference to Young [22, 53], Jung et al. [25], Saidel [68], Shigeno et al. [24], Robertson et al. [43] and Shigeno \& Ragsdale [52]. Relative volumes of the different structures derived from anatomical annotations of three samples are shown in Fig. 3.

Providing soft tissue contrast is the forte of MRI in both clinical and pre-clinical settings. In the octopus good anatomical delineation at meso-scale is shown in Fig. 1, 2. Twenty-five distinct anatomical structures have been delineated in three dimensions as shown in Fig. 2E 2G. 3D segmentation allows quantitative analy- sis, such as comparison of structure volumes across samples (Fig. 3). As expected, relative volume of brain lobes is constant across samples in these mature specimens. Lobe volumes also agree with those determined earlier $[36,69,70]$.

Streamlines are used to visualize how the anisotropy of water diffusion extends from one voxel to the next [71]. Given sufficient data (scans with different diffusion weighting - directions and/or b-values), the orientational distribution function (ODF) is used to model water diffusion at the voxel level $[57,58]$. Local orientation information is then propagated from one voxel to the next by a variety of techniques which basically track the most probable path of diffusion from a given starting point. In the mammalian brain these streamlines are straightforwardly correlated with myelinated bundles of axons $[62,72-74]$. Lack of myelin in the cephalopod 

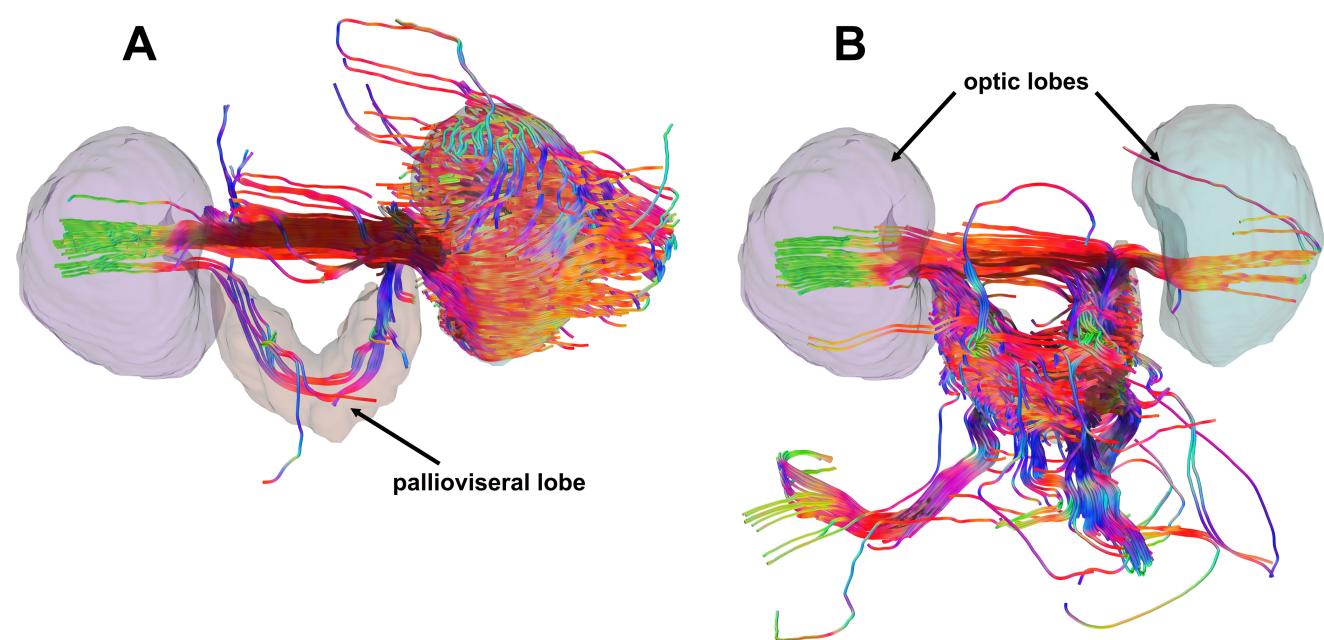

Fig. 4. Connectivity from High Angle Resolved Diffusion Imaging (HARDI) data. Streamlines from HARDI analysis originating in left optic and palliovisceral lobe (PV). Anterior view with PV and both optic lobes shown as semitransparent volumes. (A) Tracts originating in left optic bulb transverse the optic tract, connect with the contralateral lobe and enter/transverse the sub-esophageal PV also connecting with the contralateral optic lobe. (B) Tracts originating in the palliovisceral lobe connect with both optic lobes, the optic commissure, funnel and brachial nerves.

brain complicates the interpretation of streamlines, but comparison with extensive histology and anatomical MRI scans indicates that diffusion MRI tractography represents neuronal connectivity at mesoscale in the cephalopod [45].

Fig. 4 shows results of a deterministic fiber tracking algorithm [62] where the seeding region was placed in the left optic lobe (Fig. 4A) or the palliovisceral lobe (Fig. 4B). The left optic lobe streamlines connect with the optic commissure, the contralateral lobe and enter/transverse the sub esophageal palliovisceral lobe. Streamlines originating in the palliovisceral lobe connect to both optic lobes, the optic commissure and brachial funnel nerves.

Fig. 5 shows streamlines from five structures in different regions of the octopus brain: vertical lobes located in the posterior dorsal supra esophageal region; dorsal basal lobe located in the posterior basal supra-esophageal region; inferior frontal lobe located in the anterior dorsal supra-esophageal region; and brachial lobe located in the anterior basal sub-esophageal region. The vertical lobe system is thought to serve learning and memory functions analogous to the mammalian hippocampus and cerebellum $[15,52]$. Thus, although many studies find multiple connections between the vertical lobes and other structures, in this work no streamlines emerged from the vertical lobes. We attribute this to the small size of cells within the lobes and the overall small size of the lobes compared to the spatial resolution of the scans. Even the most prominent tract connecting the medial vertical lobe to the superior frontal lobe (see figure 10.5 in Young [22]) is less than 50 microns in diameter. Thus, any diffusional anisotropy due to this structure will be severely diluted in the $125 \times 125 \times 125$ micron volume element of our MR images and few if any streamlines will be observed. In contrast to the vertical lobes, the brachial lobe (Fig. 5E) has many streamlines to the arms where the central nerves are quite large in comparison to the voxel size and exhibit anisotropic water diffusion, leading to numerous streamlines.

MRI has been employed to investigate structure and function in several cephalopods, including mollusks [75], cuttlefish [47, 76], squid $[45,47,77-79]$ and octopus $[46,80]$. In most cases MRI is used to explore gross anatomy noninvasively in three dimensions for comparison with histological sections. Chung et al. [45] take a qualitatively different approach where they augment high resolution $(80 \mu \mathrm{m}$ isotropic) anatomical MRI with HARDI to produce a meso-scale connectome of the squid brain that they compare with classical and novel histologically stained samples. Interestingly, streamlines derived from the HARDI data indicate several connections between structures not previously recognized. In this work we also use anatomical and HARDI MRI to examine the significantly larger octopus brain at somewhat coarser spatial resolution.

The octopus is a visual creature with advanced learning and memory capabilities, using its complex eyes to hunt prey and avoid predators $[11,21,36]$. The optic lobes are perhaps the most prominent feature of the octopus brain, occupying a third of the brain volume [22, 51]. They are important in visual analysis and processing, learning and memory, and higher motor control. Optic lobe connections found by Young and others $[31,54,68,81]$ are also apparent in the HARDI derived streamlines. Fig. 4 illustrates the extensive connections flowing out of the optic lobe along the 


\section{side}

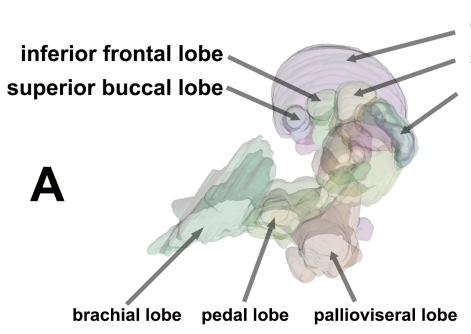

B
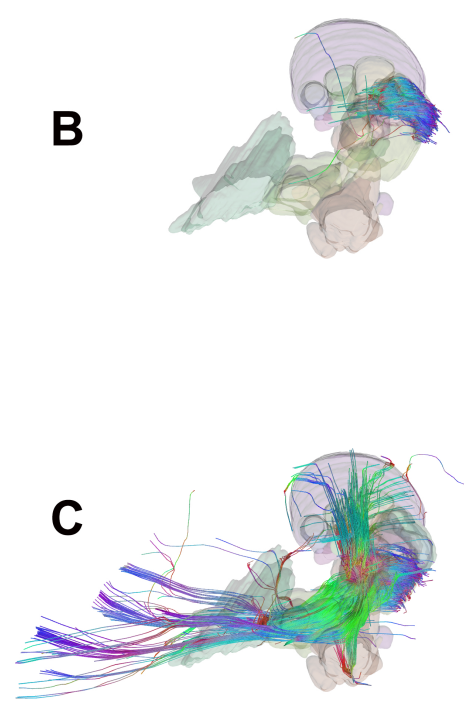

D
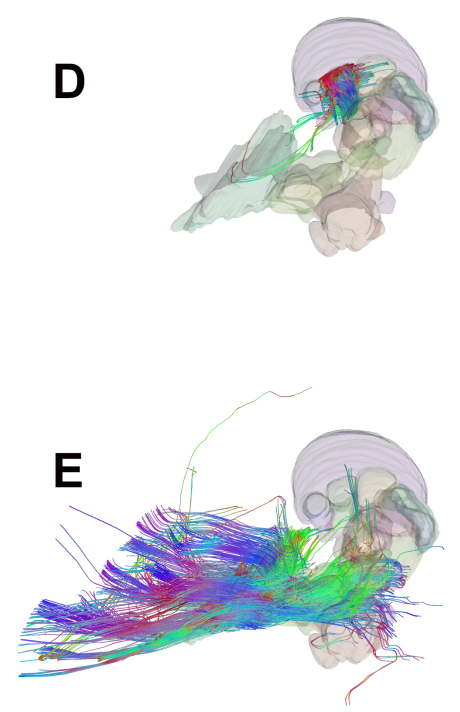

top

optic lobe

superior frontal lobe

vertical lobes
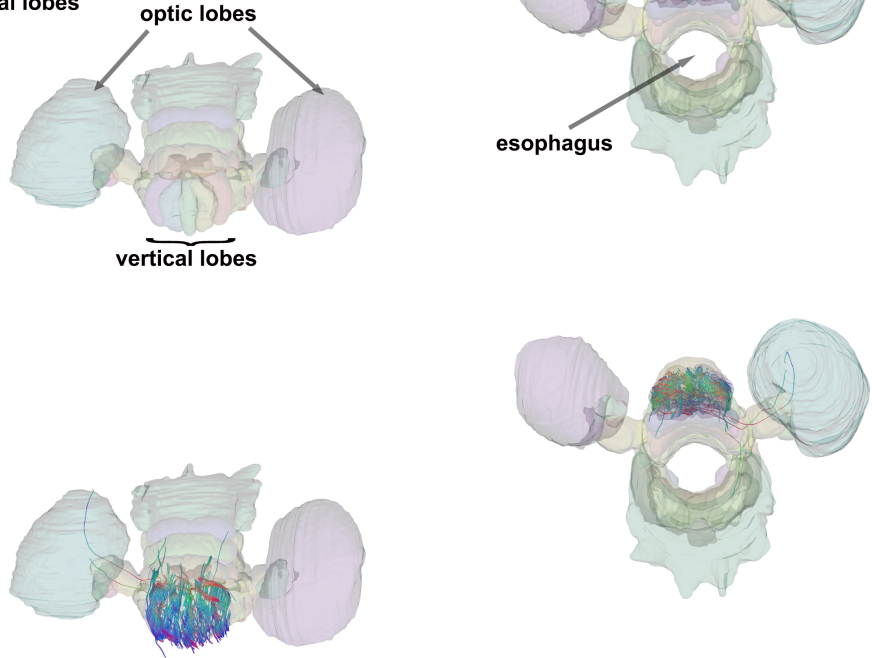

front

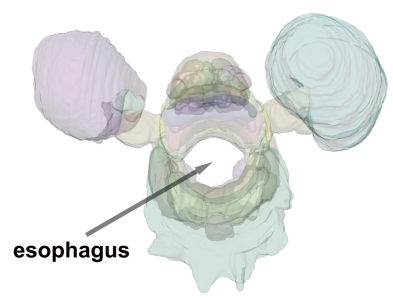

vertical lobes
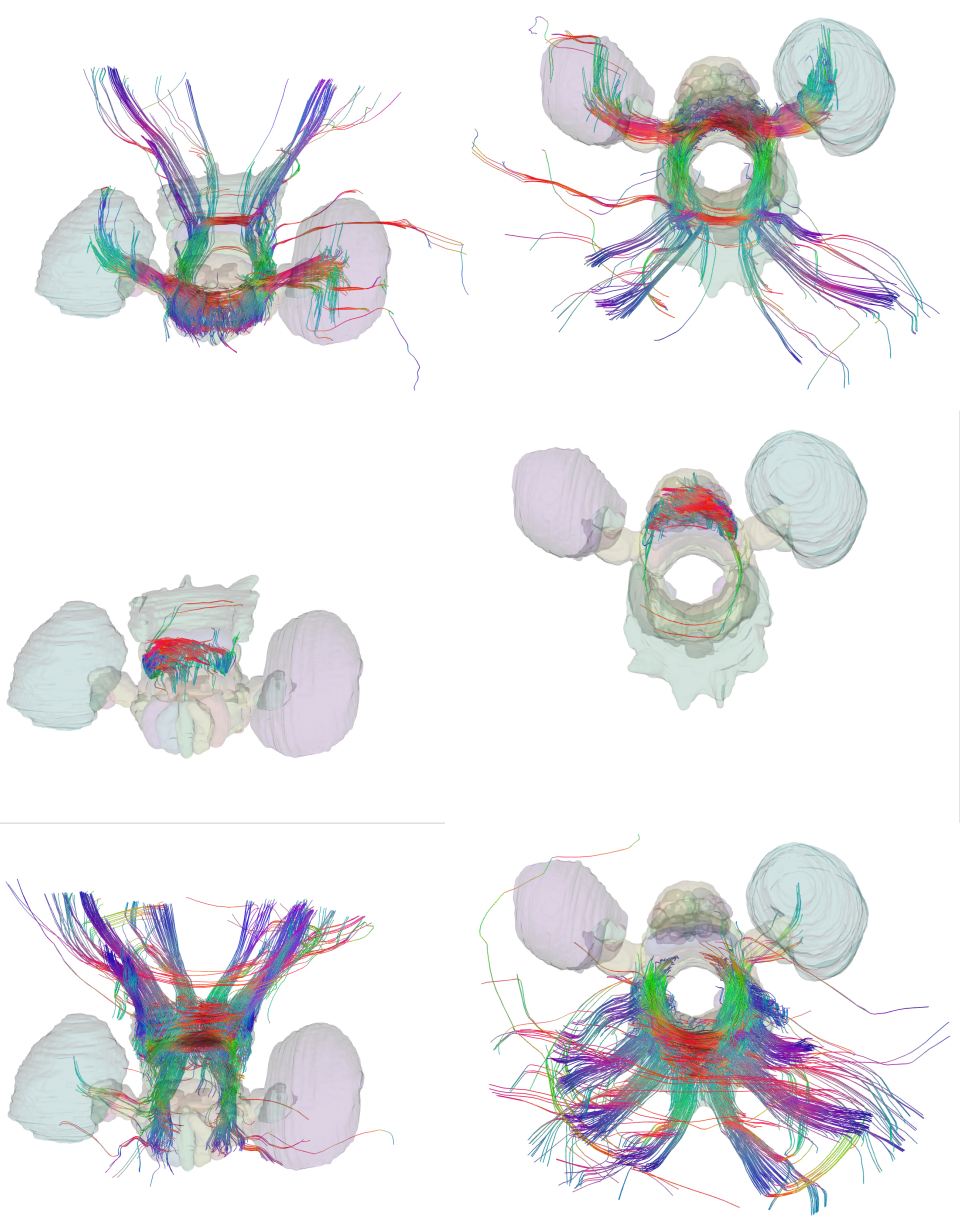

Fig. 5. Semitransparent views of streamlines originating in four different parts of the octopus brain. (A) Structures labeled in semitransparent views with no streamlines; (B) streamlines from the vertical lobes, (C) streamlines from the dorsal basal lobe; (D) streamlines from the inferior frontal lobe; and (E) streamlines from the brachial lobe. Side, top and front views are shown in columns from left to right. 
optic commissure to the contralateral lobe and down to supraesophageal structures such as the palliovisceral lobe, funnel and brachial nerves. Penetration of optic chiasm streamlines into the optic lobes are apparent in both lobes (Fig. 4, supplemental videos) and terminate mid-medulla. Streamlines within the medulla of the optic lobe are also well organized (Fig. 6) as was observed in the squid by Chung et al. [45]. Unlike Chung et al. [45] who observe a clear grid-like retinotopic network in the medulla of the squid, here tractography shows a streaming across the medulla with individual streamlines terminating within the medulla in no regular pattern (Fig. 6C, Supplemental videos). Whether this is due to difference between the species or because the lower resolution of the octopus images precludes observation of the grid-like structure re-

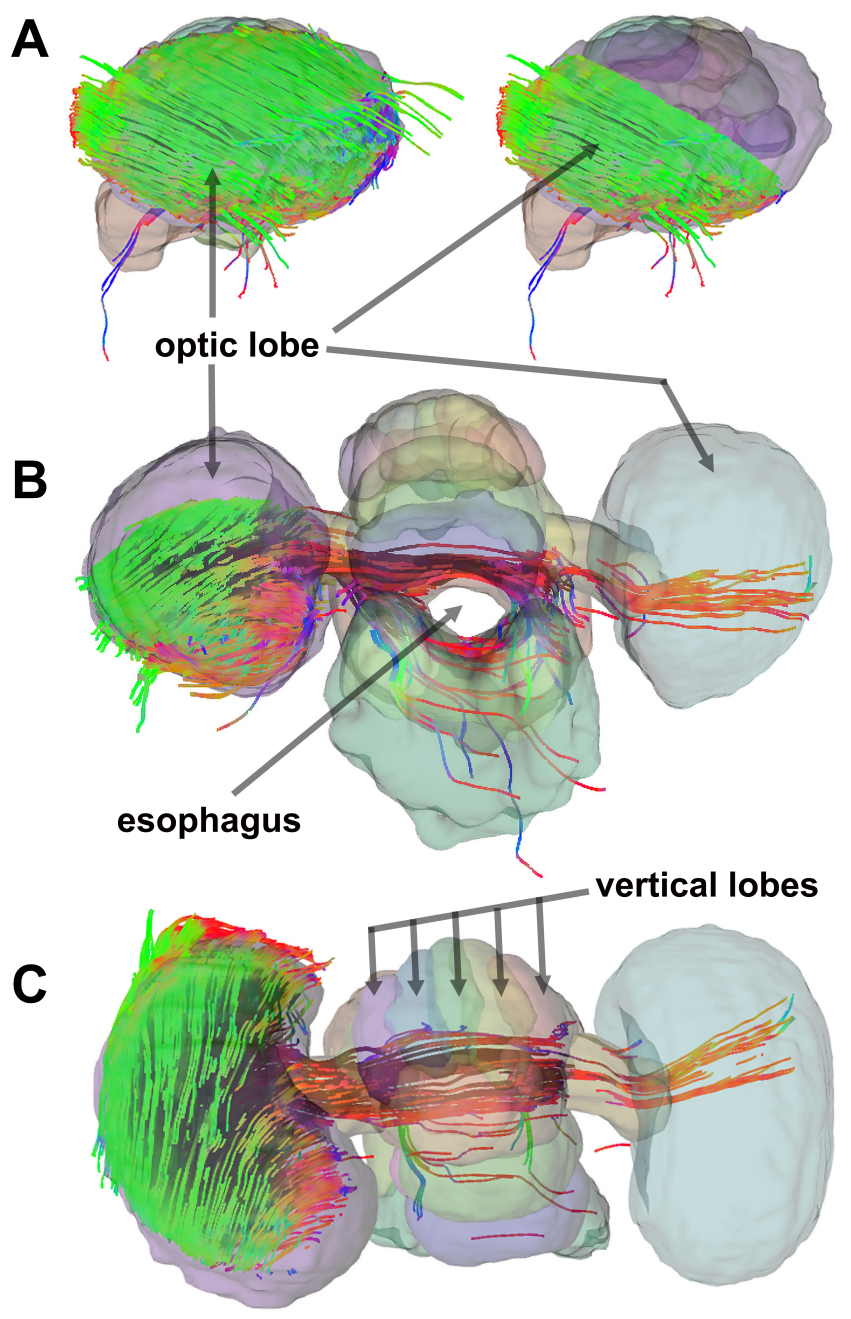

Fig. 6. Internal structure of the optic lobe. Streamlines originating in right optic lobe show the same connections as seen in Fig. 4 for the left optic lobe. (A) Side view with all streamlines (left) \& with only those below a $30^{\circ}$ cutting plane. With this deletion, the front (panel B) \& top (panel C) views show well organized roughly parallel streamlines within the optic lobe. See Supplemental Data for videos. mains to be seen. In any case, the arrangement of connection from the plexiform zone to the inner medulla is not amorphous.

Seeding the whole brain and analyzing the number of streamlines connecting structures provides a connectivity matrix $[72,73$, 82, 83], as shown in Fig. 7. The same information can be shown as chord diagrams as seen in Fig. 8. Connections between different structures culled from the literature $[12,15,23,32,33,35,43$, $52,54,68]$ are shown for comparison. Connectivity matrices and chord diagrams provide concise visual representations of complex relationships. It should be remembered that connectivities derived from diffusion MRI [73] are not precisely equivalent to tracing of neuronal axons from one structure to another that occurs with histologically stained sections [23] or where neurons are injected with dye $[43,68]$. In this analysis of diffusion images, two structures are considered connected if a sufficient number of diffusion streamlines pass through both structures, i.e., CSI $>1.5$. The connectivity matrix shown in Fig. 7 compares connectivity relationships noted in the extensive octopus literature with those derived from HARDI streamlines. We use the nomenclature of Jung et al. [25]. The preponderance of HARDI streamline connections are also described in the literature: green squares in Fig. 7. Those literature connections not found by streamlines are principally vertical lobe to other supra-esophageal lobes and between sub-esophageal lobes. Diffusion streamline connections not found in the literature (blue squares, Fig. 7) are principally found between the more basal supra-esophageal lobes, e.g., frI, subF, bucS, bucP, ba \& bd. These structures are involved in chemo-tactile memory [54] and as relay center for connections to lower motor centers [24,31]. Thus, they are somewhat analogous to the vertebrate thalamus and basal ganglia. Increased connections may indicate that more processing takes place among these lobes than previously realized.

We note that diffusion MRI work by Chung et al. [45] in the squid found significantly more streamline connections between structures than revealed in this octopus study - a factor of 4 . We attribute this to difference in how the MRI data was acquired as well as how streamlines were calculated. MRI scans for the extracted squid brains were acquired at 80 micron resolution as opposed to 125 microns for the octopus brains in this study - a volume ratio of 3.8. For similarly sized structures we expect on the order of 4 times as many streamlines in the squid as opposed to octopus data. Moreover, Chung et al. used 10 streamline seeds per voxel in their calculations while in this work 2 seeds per voxel were used. Thus, it is not surprising that the squid connectivity matrix of Chung et al. reveals significantly more connections than seen in Fig. 7 for the octopus. Moreover, we cannot rule out the notion that details of the connections in the decapod differ from the octopod.

The meso-scale nature of MRI, with spatial resolution limited 


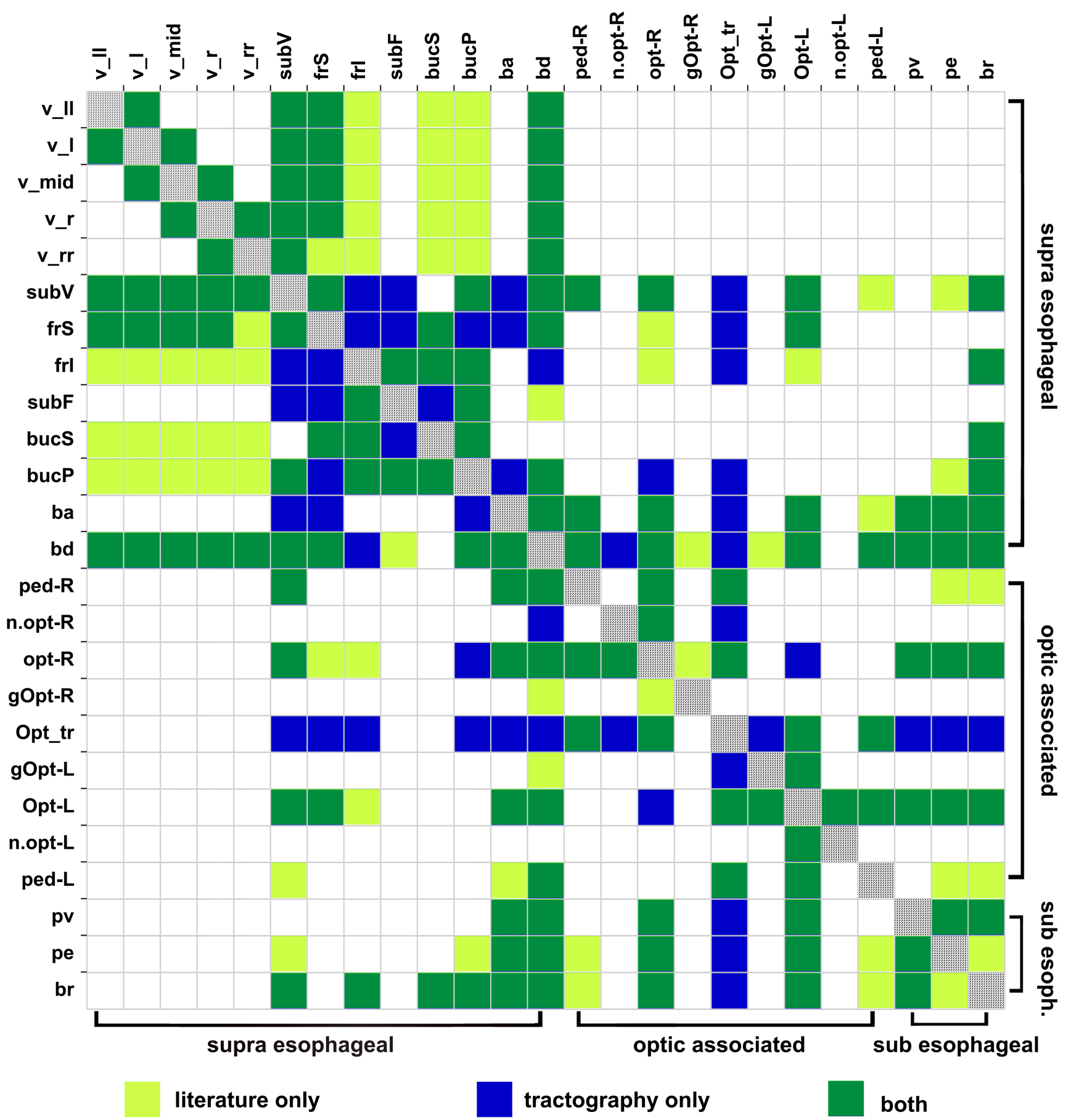

Fig. 7. Connectivity matrix comparison of neuronal connections. Yellow blocks: described in the literature but not fund by HARDI; blue blocks: found by HARDI streamline connections but not in literature; and green blocks: connections found in both. For HARDI connectivity we follow Chung et al., defining a Connectivity Strength Index (CSI) for each ROI as the median of $\log _{10}$ (count of connecting tracts) of the 3 sample. Paired regions with a CSI $>1.5$ are considered to be connected. (A) Literature; (B) tractography. Abbreviations as in Fig. 1.

to 10 s to 100 s of microns, results in loss in detail compared with optical methods. Moreover, lack of myelination in the cephalopod brain implies decreased diffusion anisotropy as compared to the vertebrate. These considerations conspire to make diffusion streamlines less than faithfully reflect connections found by histological examination. The three-dimensional nature of MRI makes it ideally suited to tracing the intrinsically three-dimensional connections found in complex brains. MRI tractography is just begin- 

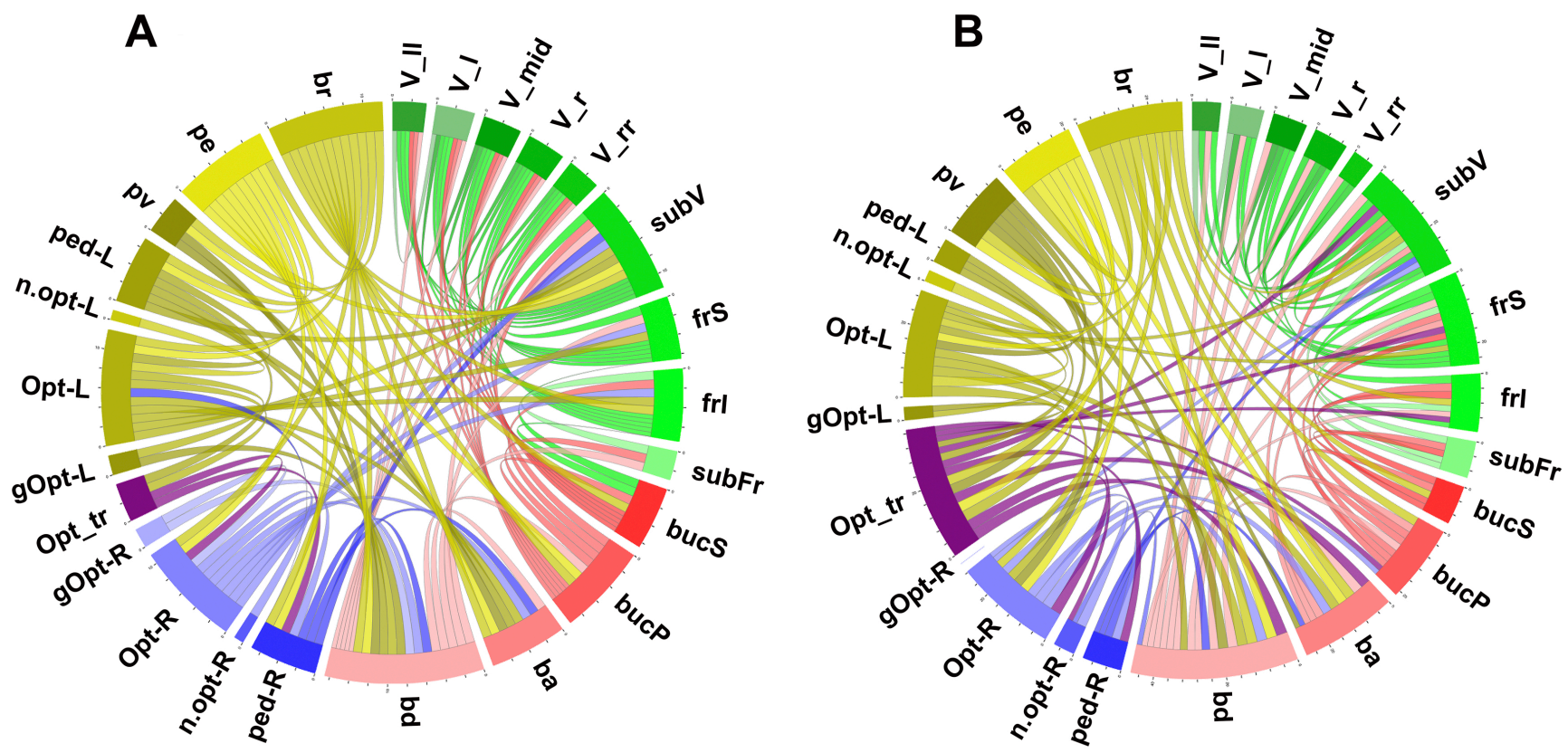

Fig. 8. Chord diagram comparison of neuronal connections. Chord diagram visualization of connectivity use the same information as connectivity matrices in Fig. 7. (A) Literature; (B) tractography with CSI>1.5. Interactive diagrams found in Supplemental Data. Abbreviations as in Fig. 1.

ning to provide insight into brain function and dysfunction in the human [84]. Similarly, insight into the intelligent behavior of the octopus will require detailed examination of connections in the cephalopod brain under different conditions (e.g. highly trained versus naïve), with and without neurochemical intervention [85, 86], at different ages, and comparison across different species (e.g. coastal versus benthonic [87]).

\section{ACKNOWLEDGEMENTS}

We gratefully acknowledge financial support from the Zilkha Neurogenetics Institute. Chuck Winkler provided invaluable assistance by collecting samples and providing cogent comments on cephalopod handling and care. Frank Yeh answered many questions about details of DSI software. Robyn Crook and Erin Baker were instrumental in providing insights into octopus neurobiology and neuroanatomy.

\section{REFERENCES}

1. Kröger B, Vinther J, Fuchs D (2011) Cephalopod origin and evolution: a congruent picture emerging from fossils, development and molecules: extant cephalopods are younger than previously realised and were under major selection to become agile, shell-less predators. Bioessays 33:602-613.

2. Strugnell J, Jackson J, Drummond AJ, Cooper A (2006) Diver- gence time estimates for major cephalopod groups: evidence from multiple genes. Cladistics 22:89-96.

3. Gutnick T, Kuba MJ (2018) Animal behavior: socializing octopus. Curr Biol 28:R1147-R1149.

4. Wang ZY, Ragsdale CW (2017) Maternal behavior and death in the octopus. Integr Comp Biol 57(Suppl 1):e440.

5. Scheel D, Godfrey-Smith P, Lawrence M (2016) Signal use by octopuses in agonistic interactions. Curr Biol 26:377-382.

6. Caldwell RL, Ross R, Rodaniche A, Huffard CL (2015) Behavior and body patterns of the Larger Pacific Striped Octopus. PLoS One 10:e0134152.

7. Hochner B, Shomrat T (2013) The neurophysiological basis of learning and memory in advanced invertebrates: the octopus and the cuttlefish. In: Handbook of behavioral neuroscience (Menzel R, Benjamin PR, eds), pp 303-317. Elsevier, London, UK.

8. Hochner B (2008) Octopuses. Curr Biol 18:R897-R898.

9. Hochner B, Shomrat T, Fiorito G (2006) The octopus: a model for a comparative analysis of the evolution of learning and memory mechanisms. Biol Bull 210:308-317.

10. Scheel D, Chancellor S, Hing M, Lawrence M, Linquist S, Godfrey-Smith P (2017) A second site occupied by Octopus tetricus at high densities, with notes on their ecology and behavior. Mar Freshw Behav Physiol 50:285-291.

11. Hanlon RT, Messenger JB (2018) Cephalopod behaviour. 2nd ed. Cambridge University Press, Cambridge, UK. 
12. Wang ZY, Ragsdale CW (2019) Cephalopod nervous system organization. In: Oxford research encyclopedia of neuroscience (Sherman SM, ed) [Internet]. Oxford University Press, New York, NY. Available from: https://www.worldcat. org/title/oxford-research-encyclopedia-of-neuroscience/ oclc/1258263320\&referer=brief_results

13. Zarrella I, Ponte G, Baldascino E, Fiorito G (2015) Learning and memory in Octopus vulgaris: a case of biological plasticity. Curr Opin Neurobiol 35:74-79.

14. Yoshida MA, Ogura A, Ikeo K, Shigeno S, Moritaki T, Winters GC, Kohn AB, Moroz LL (2015) Molecular evidence for convergence and parallelism in evolution of complex brains of cephalopod molluscs: insights from visual systems. Integr Comp Biol 55:1070-1083.

15. Shomrat T, Turchetti-Maia AL, Stern-Mentch N, Basil JA, Hochner B (2015) The vertical lobe of cephalopods: an attractive brain structure for understanding the evolution of advanced learning and memory systems. J Comp Physiol A Neuroethol Sens Neural Behav Physiol 201:947-956.

16. Hochner B (2010) Functional and comparative assessments of the octopus learning and memory system. Front Biosci (Schol Ed) 2:764-771.

17. Albertin CB, Simakov O, Mitros T, Wang ZY, Pungor JR, Edsinger-Gonzales E, Brenner S, Ragsdale CW, Rokhsar DS (2015) The octopus genome and the evolution of cephalopod neural and morphological novelties. Nature 524:220-224.

18. Edsinger E, Dölen G (2018) A conserved role for serotonergic neurotransmission in mediating social behavior in octopus. Curr Biol 28:3136-3142.e4.

19. Liscovitch-Brauer N, Alon S, Porath HT, Elstein B, Unger R, Ziv T, Admon A, Levanon EY, Rosenthal JJC, Eisenberg E (2017) Trade-off between transcriptome plasticity and genome evolution in cephalopods. Cell 169:191-202.e11.

20. Garrett S, Rosenthal JJ (2012) RNA editing underlies temperature adaptation in $\mathrm{K}+$ channels from polar octopuses. Science 335:848-851.

21. Darmaillacq AS, Dickel L, Mather J (2014) Cephalopod cognition. Cambridge University Press, Cambridge, UK.

22. Young JZ (1971) The anatomy of the nervous system of $O c$ topus vulgaris. Clarendon Press, Oxford, UK.

23. Budelmann BU, Young JZ (1985) Central pathways of the nerves of the arms and mantle of Octopus. Phil Trans R Soc Lond 310:109-122.

24. Shigeno S, Andrews PLR, Ponte G, Fiorito G (2018) Cephalopod brains: an overview of current knowledge to facilitate comparison with vertebrates. Front Physiol 9:952.

25. Jung SH, Song HY, Hyun YS, Kim YC, Whang I, Choi TY, Jo S
(2018) A brain atlas of the long arm octopus, octopus minor. Exp Neurobiol 27:257-266.

26. Levy G, Hochner B (2017) Embodied organization of $O c$ topus vulgaris morphology, vision, and locomotion. Front Physiol 8:164.

27. Winters GC, Kohn AB, Laux R, Stern N, Bostwick C, Di Cosmo A, Hochner B, Moroz LL (2016) Molecular organization of octopus brains: first insights into unique memory center signaling. Integr Comp Biol 56 (Suppl 1):e241.

28. Wild E, Wollesen T, Haszprunar G, Heß M (2015) Comparative 3D microanatomy and histology of the eyes and central nervous systems in coleoid cephalopod hatchlings. Org Divers Evol 15:37-64.

29. Pungor JR, Albertin CB, Kang S, Ragsdale CW (2013) Molecular characterization of the octopus visual system. Integr Comp Biol 53 (Suppl 1):e354.

30. Mather JA, Kuba MJ (2013) The cephalopod specialties: complex nervous system, learning, and cognition. Can J Zool 91:431-449.

31. Zullo L, Sumbre G, Agnisola C, Flash T, Hochner B (2009) Nonsomatotopic organization of the higher motor centers in octopus. Curr Biol 19:1632-1636.

32. Shomrat T, Zarrella I, Fiorito G, Hochner B (2008) The octopus vertical lobe modulates short-term learning rate and uses LTP to acquire long-term memory. Curr Biol 18:337-342.

33. Shigeno S, Parnaik R, Ragsdale CW (2008) Octopus brains: the molecular architecture of functionally identified neural systems (mollusca). J Morphol 269:1466-1467.

34. Jaaro H, Fainzilber M (2006) Building complex brains--missing pieces in an evolutionary puzzle. Brain Behav Evol 68:191195.

35. Williamson R, Chrachri A (2004) Cephalopod neural networks. Neurosignals 13:87-98.

36. Nixon M, Young JZ (2003) The brains and lives of cephalopods. Oxford University Press, New York, NY.

37. Sakurai Y, Ikeda Y (2019) Development of a contrastenhanced micro computed tomography protocol for the oval squid (Sepioteuthis lessoniana) brain. Microsc Res Tech 82:1941-1952.

38. Kerbl A, Handschuh S, Nödl MT, Metscher B, Walzl M, Wanninger A (2013) Micro-CT in cephalopod research: investigating the internal anatomy of a sepiolid squid using a nondestructive technique with special focus on the ganglionic system. J Exp Mar Biol Ecol 447:140-148.

39. Minnocci A, Cianchetti M, Mazzolai B, Sebastiani L, Laschi C (2015) Cryo-scanning electron microscopy investigation of the Octopus vulgaris arm structures for the design of an 
octopus-like arm artefact. Microsc Res Tech 78:1133-1145.

40. Gray EG, Young JZ (1969) Electron microscopy of the gliovascular organization of the brain of Octopus. Phil Trans R Soc Lond B 255:13-32.

41. Zullo L, Buschiazzo A, Massollo M, Riondato M, Democrito A, Marini C, Benfenati F, Sambuceti G (2018) Small-animal ${ }^{18}$ F-FDG PET for research on Octopus vulgaris: applications and future directions in invertebrate neuroscience and tissue regeneration. J Nucl Med 59:1302-1307.

42. Wollesen T, Loesel R, Wanninger A (2009) Pygmy squids and giant brains: mapping the complex cephalopod CNS by phalloidin staining of vibratome sections and whole-mount preparations. J Neurosci Methods 179:63-67.

43. Robertson JD, Schwartz OM, Lee P (1993) Carbocyanine dye labeling reveals a new motor nucleus in octopus brain. J Comp Neurol 328:485-500.

44. Imperadore P, Lepore MG, Ponte G, Pflüger HJ, Fiorito G (2019) Neural pathways in the pallial nerve and arm nerve cord revealed by neurobiotin backfilling in the cephalopod mollusk Octopus vulgaris. Invert Neurosci 19:5.

45. Chung WS, Kurniawan ND, Marshall NJ (2020) Toward an MRI-based mesoscale connectome of the squid brain. iScience 23:100816.

46. Jiang X, Lu H, Shigeno S, Tan LH, Yang Y, Ragsdale CW, Gao JH (2014) Octopus visual system: a functional MRI model for detecting neuronal electric currents without a blood-oxygenlevel-dependent confound. Magn Reson Med 72:1311-1319.

47. Quast MJ, Neumeister H, Ezell EL, Budelmann BU (2001) MR microscopy of cobalt-labeled nerve cells and pathways in an invertebrate brain (Sepia officinalis, Cephalopoda). Magn Reson Med 45:575-579.

48. Blackband SJ, Stoskopf MK (1990) In vivo nuclear magnetic resonance imaging and spectroscopy of aquatic organisms. Magn Reson Imaging 8:191-198.

49. Allan Johnson G, Wang N, Anderson RJ, Chen M, Cofer GP, Gee JC, Pratson F, Tustison N, White LE (2019) Whole mouse brain connectomics. J Comp Neurol 527:2146-2157.

50. Oh SW, Harris JA, Ng L, Winslow B, Cain N, Mihalas S, Wang Q, Lau C, Kuan L, Henry AM, Mortrud MT, Ouellette B, Nguyen TN, Sorensen SA, Slaughterbeck CR, Wakeman W, Li Y, Feng D, Ho A, Nicholas E, Hirokawa KE, Bohn P, Joines KM, Peng H, Hawrylycz MJ, Phillips JW, Hohmann JG, Wohnoutka P, Gerfen CR, Koch C, Bernard A, Dang C, Jones AR, Zeng H (2014) A mesoscale connectome of the mouse brain. Nature 508:207-214.

51. Young JZ (1963) The number and sizes of nerve cells in Octopus. Proc Zool Soc Lond 140:229-254.
52. Shigeno S, Ragsdale CW (2015) The gyri of the octopus vertical lobe have distinct neurochemical identities. J Comp Neurol 523:1297-1317.

53. Young JZ (1988) Octopus brain. In: Comparative neuroscience and neurobiology (Irwin LN, ed), pp 97-99. Birkhäuser, Boston, MA.

54. Young JZ (1983) The distributed tactile memory system of Octopus. Proc R Soc Lond 218:135-176.

55. Butler-Struben HM, Brophy SM, Johnson NA, Crook RJ (2018) In vivo recording of neural and behavioral correlates of anesthesia induction, reversal, and euthanasia in cephalopod molluscs. Front Physiol 9:109.

56. Pugliese C, Mazza R, Andrews PL, Cerra MC, Fiorito G, Gattuso A (2016) Effect of different formulations of magnesium chloride used as anesthetic agents on the performance of the isolated heart of Octopus vulgaris. Front Physiol 7:610.

57. Mori S, Tournier JD (2014) Chapter 8 - moving beyond DTI: high angular resolution diffusion imaging (HARDI). In: Introduction to diffusion tensor imaging (Mori S, Tournier JD, eds), 2nd ed, pp 65-78. Academic Press, San Diego, CA.

58. Daianu M, Jacobs RE, Weitz TM, Town TC, Thompson PM (2015) Multi-shell hybrid diffusion imaging (HYDI) at 7 Tesla in TgF344-AD transgenic Alzheimer rats. PLoS One 10:e0145205.

59. Caruyer E, Lenglet C, Sapiro G, Deriche R (2013) Design of multishell sampling schemes with uniform coverage in diffusion MRI. Magn Reson Med 69:1534-1540.

60. Yeh FC, Liu L, Hitchens TK, Wu YL (2017) Mapping immune cell infiltration using restricted diffusion MRI. Magn Reson Med 77:603-612.

61. Yeh FC, Wedeen VJ, Tseng WY (2010) Generalized q-sampling imaging. IEEE Trans Med Imaging 29:1626-1635.

62. Yeh FC, Verstynen TD, Wang Y, Fernández-Miranda JC, Tseng WY (2013) Deterministic diffusion fiber tracking improved by quantitative anisotropy. PLoS One 8:e80713.

63. Joachimiak MP, Weisman JL, May BCh (2006) JColorGrid: software for the visualization of biological measurements. BMC Bioinformatics 7:225.

64. Flor M (2020) chorddiag 0.1.3 [Internet]. GitHub, San Francisco, CA. Available from: https://github.com/mattflor/chorddiag/.

65. Rorden C, Brett M (2000) Stereotaxic display of brain lesions. Behav Neurol 12:191-200.

66. Schindelin J, Arganda-Carreras I, Frise E, Kaynig V, Longair M, Pietzsch T, Preibisch S, Rueden C, Saalfeld S, Schmid B, Tinevez JY, White DJ, Hartenstein V, Eliceiri K, Tomancak P, Cardona A (2012) Fiji: an open-source platform for biologi- 
cal-image analysis. Nat Methods 9:676-682.

67. Koizumi M, Shigeno S, Mizunami M, Tanaka NK (2016) Three-dimensional brain atlas of pygmy squid, Idiosepius paradoxus, revealing the largest relative vertical lobe system volume among the cephalopods. J Comp Neurol 524:21422157.

68. Saidel WM (1982) Connections of the octopus optic lobe: an HRP study. J Comp Neurol 206:346-358.

69. Maddock L, Young JZ (1987) Quantitative differences among the brains of cephalopods. J Zool 212:739-767.

70. Wirz K (1959) [Biometric study of the nervous system of cephalopods]. Bull Biol 93:78-117. French.

71. Mori S, Tournier JD (2014) Chapter 9 - Fiber-Tracking: 3-dimensional tract reconstruction. In: Introduction to diffusion tensor imaging (Mori S, Tournier JD, eds), 2nd ed, pp 79-96. Academic Press, San Diego, CA.

72. Yeh CH, Jones DK, Liang X, Descoteaux M, Connelly A (2021) Mapping structural connectivity using diffusion MRI: challenges and opportunities. J Magn Reson Imaging 53:16661682.

73. Sotiropoulos SN, Zalesky A (2019) Building connectomes using diffusion MRI: why, how and but. NMR Biomed 32:e3752.

74. Merlet S, Caruyer E, Ghosh A, Deriche R (2013) A computational diffusion MRI and parametric dictionary learning framework for modeling the diffusion signal and its features. Med Image Anal 17:830-843.

75. Ziegler A, Bock C, Ketten DR, Mair RW, Mueller S, Nagelmann N, Pracht ED, Schröder L (2018) Digital three-dimensional imaging techniques provide new analytical pathways for malacological research. Am Malacol Bull 36:248-273.

76. Liu YC, Liu TH, Su CH, Chiao CC (2017) Neural organization of the optic lobe changes steadily from late embryonic stage to adulthood in cuttlefish Sepia pharaonis. Front Physiol 8:538.

77. Liu YC, Chung WS, Yu CC, Hsu ST, Chan FL, Liu TH, Su CH, Hwu Y, Marshall NJ, Chiao CC (2018) Morphological changes of the optic lobe from late embryonic to adult stages in oval squids Sepioteuthis lessoniana. J Morphol 279:75-85.

78. Chung WS, Marshall NJ (2017) Complex visual adaptations in squid for specific tasks in different environments. Front Physiol 8:105.

79. Melzner F, Bock C, Pörtner HO (2007) Coordination between ventilatory pressure oscillations and venous return in the cephalopod Sepia officinalis under control conditions, spontaneous exercise and recovery. J Comp Physiol B 177:117.

80. Ziegler A, Sagorny C (2021) Holistic description of new deep sea megafauna (Cephalopoda: Cirrata) using a minimally invasive approach. BMC Biol 19:81.

81. Young JZ (1995) Multiple matrices in the memory system of octopus. In: Cephalopod neurobiology: neuroscience studies in squid, octopus and cuttlefish (Joan Abbott N, Williamson R, Maddock L, eds), pp 431-443. Oxford University Press, New York, NY.

82. Fornito A, Zalesky A, Bullmore ET (2016) Chapter 3 - connectivity matrices and brain graphs. In: Fundamentals of brain network analysis (Fornito A, Zalesky A, Bullmore ET, eds), pp 89-113. Academic Press, San Diego, CA.

83. Jeurissen B, Descoteaux M, Mori S, Leemans A (2019) Diffusion MRI fiber tractography of the brain. NMR Biomed 32:e3785.

84. Forkel SJ, Friedrich P, Thiebaut de Schotten M, Howells H (2022) White matter variability, cognition, and disorders: a systematic review. Brain Struct Funct 227:529-544.

85. De Lisa E, Paolucci M, Di Cosmo A (2012) Conservative nature of oestradiol signalling pathways in the brain lobes of Octopus vulgaris involved in reproduction, learning and motor coordination. J Neuroendocrinol 24:275-284.

86. Perez PV, Butler-Struben HM, Crook RJ (2017) The selective serotonin reuptake inhibitor fluoxetine increases spontaneous afferent firing, but not mechanonociceptive sensitization, in octopus. Invert Neurosci 17:10.

87. Chung WS, Kurniawan ND, Marshall NJ (2022) Comparative brain structure and visual processing in octopus from different habitats. Curr Biol 32:97-110.e4. 